\title{
Bictegravir combined with emtricitabine and tenofovir alafenamide versus dolutegravir, abacavir, and lamivudine for initial treatment of HIV-1 infection: week 96 results from a randomised, double-blind, multicentre, phase 3, non-inferiority trial
}

\author{
David A Wohl, Yazdan Yazdanpanah, Axel Baumgarten, Amanda Clarke, Melanie A Thompson, Cynthia Brinson, Debbie Hagins, \\ Moti N Ramgopal, Andrea Antinori, Xuelian Wei, Rima Acosta, Sean E Collins, Diana Brainard, Hal Martin
}

\section{Summary}

Background Bictegravir co-formulated with emtricitabine and tenofovir alafenamide as a fixed-dose combination is recommended for treatment of HIV-1-infection and might be better tolerated than other integrase inhibitor-based single-tablet regimens, but long-term outcomes data are not available. We assessed the efficacy, safety and tolerability of bictegravir, emtricitabine, and tenofovir alafenamide compared with co-formulated dolutegravir, abacavir, and lamivudine at week 96.

Methods This ongoing, randomised, double-blind, multicentre, active-controlled, phase 3, non-inferiority trial was done at 122 outpatient centres in nine countries. We enrolled adults (aged $\geq 18$ years) living with HIV who were treatment naive and HLA-B $* 5701$ negative, did not have hepatitis $B$ virus infection, and had an estimated glomerular filtration rate of at least $50 \mathrm{~mL} / \mathrm{min}$. We randomly assigned participants (1:1) to receive co-formulated bictegravir $50 \mathrm{mg}$, emtricitabine $200 \mathrm{mg}$, and tenofovir alafenamide $25 \mathrm{mg}$ (the bictegravir group) or co-formulated dolutegravir $50 \mathrm{mg}$, abacavir $600 \mathrm{mg}$, and lamivudine $300 \mathrm{mg}$ (the dolutegravir group), each with matching placebo, once daily for 144 weeks. Treatment allocation was masked to all participants and investigators. All participants who received at least one dose of study drug were included in primary efficacy and safety analyses. We previously reported the primary endpoint. Here, we report the week 96 secondary outcome of proportion of participants with plasma HIV-1 RNA less than 50 copies per $\mathrm{mL}$ at week 96 by US Food and Drug Administration snapshot algorithm, with a prespecified non-inferiority margin of $-12 \%$. This study was registered with ClinicalTrials.gov, number NCT02607930.

Findings Between Nov 13, 2015, and July 14, 2016, we screened 739 participants, of whom 108 were excluded and 631 enrolled and randomly assigned to bictegravir, emtricitabine, and tenofovir alafenamide $(\mathrm{n}=316)$ or dolutegravir, abacavir, and lamivudine $(\mathrm{n}=315)$. Two participants in the bictegravir group did not receive at least one dose of their assigned drug and were excluded from analyses. At week 96, bictegravir, emtricitabine, and tenofovir alafenamide was non-inferior to dolutegravir, abacavir, and lamivudine, with $276(88 \%)$ of 314 participants in the bictegravir group versus $283(90 \%)$ of 315 participants in the dolutegravir group achieving HIV-1 RNA less than 50 copies per mL (difference $-1.9 \%$; $95 \%$ CI -6.9 to $3 \cdot 1)$. The most common adverse events were nausea (36 [11\%] of 314 for the bictegravir group vs 76 [24\%] of 315 for the dolutegravir group), diarrhoea (48 [15\%] vs 50 [16\%]), and headache (41 [13\%] vs 51 [16\%]). 36 (11\%) participants in the bictegravir group versus 39 (12\%) participants in the dolutegravir group had a serious adverse event. Two individuals died in the bictegravir group (recreational drug overdose and suicide, neither of which was treatment related) and none died in the dolutegravir group. No participants discontinued because of adverse events in the bictegravir group compared with five (2\%) of 315 in the dolutegravir group. Study drug-related adverse events were reported for $89(28 \%)$ participants in the bictegravir group and $127(40 \%)$ in the dolutegravir group.

Interpretation These week 96 data support bictegravir, emtricitabine, and tenofovir alafenamide as a safe, well tolerated, and durable treatment for people living with HIV-1 with no emergent resistance.

Funding Gilead Sciences, Inc.

\section{Introduction}

The evolution of HIV therapeutics has been marked by a shift from multiple pill regimens given two to three times per day to once-daily, single-tablet formulations with improved potency and tolerability. Two single-tablet regimens are recommended as initial
Lancet HIV 2019; 6: e355-63 Published Online May 5, 2019 http://dx.doi.org/10.1016 S2352-3018(19)30077-3 See Comment page e342 Department of Medicine, Institute of Global Health and Infectious Diseases, University of North Carolina at Chapel Hill, Chapel Hill, NC, USA (Prof D A Wohl MD); Department of Medicine, Paris Diderot University, Paris, France (ProfY Yazdanpanah MD); Zentrum für Infektiologie Berlin Prenzlauer Berg, Berlin, Germany (A Baumgarten MD) Claude Nicol Centre, Royal Sussex County Hospital, Brighton and Sussex University Hospitals NHS Trust, Brighton, UK (A Clarke MD); AIDS Research Consortium of Atlanta Atlanta, GA, USA (M A Thompson MD); Central Texas Clinical Research, Austin, TX, USA (C Brinson MD); Georgia Department of Public Health, Coastal Health District, Chatham Care Center, Savannah, GA, USA (D Hagins MD); Midway Immunology Center, Fort Pierce, FL, USA (M N Ramgopal MD); National Institute for Infectious Diseases Lazzaro SpallanzaniIRCCS, Roma, Italy (A Antinori MD); Department of Biometrics, Gilead Sciences Inc, Foster City, CA, USA (X Wei PhD); Department of Virology, Gilead Sciences Inc, Foster City, CA, USA (R Acosta BS); and Department of HIV Clinical Research, Gilead Sciences Inc, Foster City, CA, USA (S E Collins MD, D Brainard MD, H Martin MD) 
Correspondence to: Dr Sean E Collins, Gilead Sciences Inc, Foster City, CA 94404, USA sean.collins@gilead.com

\section{Research in context}

\section{Evidence before this study}

We searched PubMed on Jan 30, 2019, for randomised clinical trials of dolutegravir and bictegravir in people living with HIV-1, with title or abstract search terms of "dolutegravir" and "bictegravir" and "randomised" or "randomized". We used internet search engines to identify governmental, non-governmental, and professional medical society practice guidelines relevant to bictegravir and dolutegravir. Searches were limited to English language publications between Jan 1, 1997, and Jan 30, 2019. Our search yielded reports of week-48 outcomes from six phase 2 and phase 3 studies of bictegravir, emtricitabine, and tenofovir alafenamide in treatment-naive and treatment-experienced participants. Included in these articles were the week-48 outcomes from this clinical trial (GS-US-380-1489), which showed non-inferior efficacy and less nausea with bictegravir, emtricitabine, and tenofovir alafenamide than with dolutegravir, abacavir, and lamivudine in treatment-naive participants at that timepoint. Three other phase 3 studies showed non-inferior efficacy of bictegravir, emtricitabine, and tenofovir alafenamide and confirmed safety at week 48; one in treatment-naive participants compared with dolutegravir, emtricitabine, and tenofovir alafenamide and two in virologically suppressed participants switching to bictegravir, emtricitabine, and tenofovir alafenamide compared with continuing either dolutegravir, abacavir, and lamivudine or a protease inhibitor-based regimen. Through 48 weeks, co-formulated bictegravir, emtricitabine, and tenofovir alafenamide had a similar renal, bone, and lipid safety profile relative to most comparators, with improved fasting lipid profile for those switched away from a boosted protease inhibitor, abacavir, and lamivudine. Based on week 48 data from the phase 3 trials, co-formulated bictegravir, emtricitabine, and tenofovir alafenamide became a guidelines-recommended regimen for initial treatment of adults with HIV, although longer-term data are needed to inform clinical care.

therapy of HIV-1 infection by major treatment guideline panels: co-formulated bictegravir, emtricitabine, and tenofovir alafenamide and co-formulated dolutegravir, abacavir, and lamivudine. ${ }^{1-3}$

These regimens containing integrase strand transfer inhibitors (INSTIs) are free from pharmacological boosters, and therefore have few drug interactions and can be taken with or without food. Both are also recommended for individuals regardless of pretreatment HIV-1 RNA level or CD4 cell count. Dolutegravir, abacavir, and lamivudine cannot be used in individuals who have the HLA-B*5701 allele associated with hypersensitivity to abacavir, are co-infected with hepatitis B virus, or have an estimated glomerular filtration rate (eGFR) less than $50 \mathrm{~mL} / \mathrm{min}^{2,4}$ By contrast, bictegravir, emtricitabine, and tenofovir alafenamide can be used in such individuals and is approved for those with an eGFR greater than $30 \mathrm{~mL} / \mathrm{min}^{2,5}$

\section{Added value of this study}

This study is a randomised, double-blind phase 3 trial and among the first to provide evidence of the longer-term safety and efficacy of bictegravir, emtricitabine, and tenofovir alafenamide through 96 weeks. Compared with another guidelines-recommended treatment regimen (dolutegravir, abacavir, and lamivudine), co-formulated bictegravir, emtricitabine, and tenofovir alafenamide showed non-inferior efficacy. No participants on either regimen had virological failure with emergent resistance, further demonstrating the high barrier to resistance of the study regimens. Similar to week 48, nausea and other drug-related adverse effects were fewer in those treated with co-formulated bictegravir, emtricitabine, and tenofovir alafenamide than those treated with dolutegravir. This study provides longer-term data to support the durable efficacy and continued tolerability of bictegravir, emtricitabine, and tenofovir alafenamide, with no treatment-emergent resistance. Data from this trial also offers a continued look at the different safety profiles of these two single-tablet regimens.

\section{Implications of all the available evidence}

Data from this study complement those from another 96-week report of co-formulated bictegravir, emtricitabine, and tenofovir alafenamide versus dolutegravir with emtricitabine and tenofovir alafenamide in treatment-naive individuals. Co-formulated bictegravir, emtricitabine, and tenofovir alafenamide can be administered once daily, does not require HLA B* 5701 testing, and provides guideline-recommended therapy for people with HIV and with HIV and hepatitis B virus co-infection. The efficacy of switching to co-formulated bictegravir, emtricitabine, and tenofovir alafenamide in people with virological suppression on other antiretroviral regimens has been shown in different populations of patients. Together, these studies offer longer-term efficacy and safety data to guide treatment decisions for people living with HIV-1.

Although both regimens have been shown in clinical trials to be potent and well tolerated, they had not previously been directly compared until this randomised controlled trial, comparing these two regimens in adults living with HIV and without previous antiretroviral therapy. We previously reported the primary endpoint at 48 weeks after the start of treatment, showing that bictegravir, emtricitabine, and tenofovir alafenamide was non-inferior to dolutegravir, abacavir, and lamivudine; virological failure was rare and treatment resistance was not detected in any participants. ${ }^{6}$ However, bictegravir, emtricitabine, and tenofovir alafenamide had better gastrointestinal tolerability in adverse event reporting and patient-reported outcome questionnaires. ${ }^{6,7}$

Here, we report on the 96-week outcomes of this trial to provide longer-term data on the relative efficacy and safety, including tolerability and renal, bone, and lipid 
profiles, of these two once-daily, single-tablet, INSTIcontaining regimens in individuals without previous HIV-1 therapy.

\section{Methods}

\section{Study design and participants}

This randomised, double-blind, multicentre, activecontrolled, phase 3, non-inferiority trial was done at 122 outpatient centres in nine countries: Belgium, Canada, Dominican Republic, France, Germany, Italy, Spain, the UK, and the USA. Detailed methods have been previously published. ${ }^{6}$ Briefly, study investigators enrolled adults (aged $\geq 18$ years) with HIV- 1 infection who were treatment naive, did not have HLA-B*5701 mutation or hepatitis B virus infection, and had eGFR of at least $50 \mathrm{~mL} / \mathrm{min}$ (calculated by the Cockcroft-Gault equation). This study was done in accordance with the Declaration of Helsinki and was approved by central or site-specific review boards or ethics committees. All participants gave written informed consent.

\section{Randomisation and masking}

We randomly assigned participants (1:1) to either the bictegravir group (bictegravir, emtricitabine, and tenofovir alafenamide) or dolutegravir group (dolutegravir, abacavir, and lamivudine). Participants also received placebo tablets matching the alternative treatment. Randomisation was stratified b y H IV-1 R NA ( $\leq 100000$ c opies p er m L, 100001-400000 copies per $\mathrm{mL}$, or $>400000$ copies per $\mathrm{mL}$ ), CD4 count ( $<50$ cells per $\mu \mathrm{L}, 50-199$ cells per $\mu \mathrm{L}$, or $\geq 200$ cells per $\mu \mathrm{L}$ ), and region (in the USA or outside the USA) at screening.

\section{Procedures}

Participants were given once-daily, oral fixed-dose combination of either bictegravir $50 \mathrm{mg}$, emtricitabine $200 \mathrm{mg}$, and tenofovir alafenamide $25 \mathrm{mg}$ or dolutegravir $50 \mathrm{mg}$, abacavir $600 \mathrm{mg}$, and lamivudine $300 \mathrm{mg}$. Both regimens were given without regard to food. We did study visits at weeks 4, 8, and 12 after baseline, and every 12 weeks thereafter, with masked treatment visits planned until week 144 as previously reported. ${ }^{6}$ Laboratory tests included haematological analysis, serum chemistry tests, fasting lipids, CD4 counts, renal function (eGFR, urine albumin-tocreatinine ratio, retinol binding protein-to-creatinine ratio, $\beta 2$-microglobulin-to-creatinine ratio; Covance Laboratories, Indianapolis, IN, USA), and HIV-1 RNA plasma measurements (Roche TaqMan 2.0; Roche Diagnostics, Rotkreuz, Switzerland). Protocol-defined resistance testing (Monogram Biosciences Inc, San Francisco, CA, USA) was done for any participant who had HIV-1 RNA of at least 50 copies per mL with a confirmed HIV-1 RNA of at least 200 copies per $\mathrm{mL}$ at the consecutive visit, or who had HIV-1 RNA of at least 200 copies per $\mathrm{mL}$ at week 48 , week 96 , or the last visit on study drug after week 8 , and who did not subsequently have HIV-1 RNA less than 50 copies per $\mathrm{mL}$ while on study drug.

We did dual-energy x-ray absorptiometry scans for hip and lumbar spine bone mineral density before drug administration at baseline and then at weeks 24, 48, and 96. One centre masked to treatment group assignment read all scans (BioClinica, Newtown, PA, USA). Safety was assessed by physical examinations, laboratory tests, 12-lead electrocardiogram, concomitant drugs, and recording of adverse events, which were coded with the Medical Dictionary for Regulatory Activities (MedDRA, version 21.0). Relatedness of adverse events to blinded study drugs was assessed by the investigator in a binary manner (yes or no).

\section{Outcomes}

We previously reported the primary outcome: ${ }^{6}$ the proportion of participants who had plasma HIV-1 RNA less than 50 copies per $\mathrm{mL}$ at week 48 , as defined by the US Food and Drug Administration (FDA) snapshot algorithm. ${ }^{6,8}$ Secondary analyses of efficacy were planned for week 96 and week 144 using the same methods. Here we report the 96 week results.

We also assessed virological efficacy at week 96 in prespecified subgroups of age, sex, race, baseline HIV-1 RNA, baseline CD4 cell count, geographic region, and study medication adherence. Other secondary efficacy analyses included the proportion of participants with plasma HIV-1 RNA less than 50 copies per $\mathrm{mL}$ at week 96 when imputing missing as failure and missing as excluded data; the proportion of participants with plasma HIV-1 RNA $<20$ copies per $m L$ at week 96 by snapshot algorithm; change from baseline in CD4 cell count; and changes from baseline in hip and lumbar spine bone mineral density.

Adverse event incidence and changes in fasting lipids at week 96 were assessed by treatment group. Renal safety assessments included the change from baseline in serum creatinine and eGFR at week 96, treatment-emergent proteinuria through week 96, and percentage changes from baseline in urine retinol binding protein to creatinine ratio, urine $\beta 2$-microglobulin-to-creatinine ratio and urine albumin-to-creatinine ratio at week 96 .

\section{Statistical analysis}

Sample size justification was previously reported based on the primary outcome. ${ }^{6}$ Statistical analyses at week 96 followed the same methods previously reported for week 48. We assessed plasma HIV-1 RNA less than 50 copies per $\mathrm{mL}$ at week 96 (between days 631 and 714, inclusive) in the full analysis set (all participants who received at least one dose of their assigned study drug) either when participants had completed their week 96 study visit or had prematurely discontinued the study drug. We did a per-protocol analysis excluding participants who had measurements missing for HIV-1 RNA at week 96 (unless this was due to discontinuation because of no efficacy), low adherence (ie, adherence at or below the 


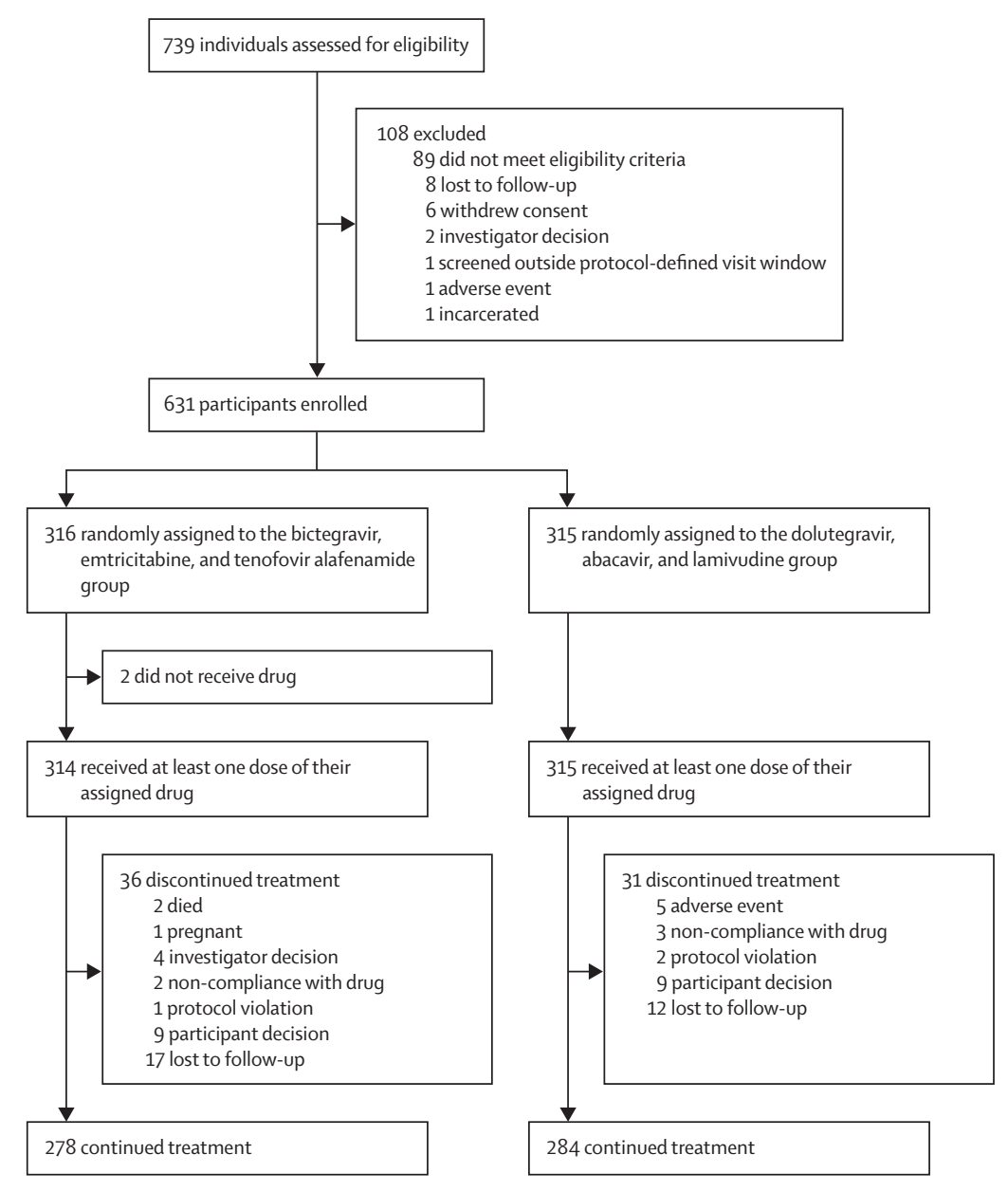

Figure 1: Trial profile through week 96

$2 \cdot 5$ th percentile among those in the study), or were taking medications prohibited by the protocol at study entry.

Change from baseline in CD4 cell count at week 96 was summarised by treatment group with descriptive statistics based on the full analysis set using observed on-treatment data.

We summarised baseline characteristics with descriptive statistics for the safety analysis set, which included all randomly assigned participants who received at least one dose of study drug. For categorical data, we calculated $\mathrm{p}$ values using the Cochran-Mantel-Haenszel test (general association statistic was used for nominal data, the row mean scores differ statistic was used for ordinal data) for treatment comparison. For continuous data, we used the two-sided Wilcoxon rank-sum test. We used Fisher's exact test for any adverse events occurring with a more than $5 \%$ difference in incidence between treatment groups. For bone mineral density data, the $\mathrm{p}$ values, the differences in percentage changes from baseline between treatment groups, and their 95\% CIs were constructed with analysis of variance model,

\begin{tabular}{|c|c|c|}
\hline & $\begin{array}{l}\text { Bictegravir, } \\
\text { emtricitabine, } \\
\text { and tenofovir } \\
\text { alafenamide } \\
\text { group }(n=314)\end{array}$ & $\begin{array}{l}\text { Dolutegravir, } \\
\text { abacavir, } \\
\text { and lamivudine } \\
\text { group }(n=315)\end{array}$ \\
\hline Age, years & $31(25-41)$ & $32(26-40)$ \\
\hline \multicolumn{3}{|l|}{ Sex } \\
\hline Women & $29(9 \%)$ & $33(10 \%)$ \\
\hline Men & $285(91 \%)$ & $282(90 \%)$ \\
\hline \multicolumn{3}{|l|}{ Race } \\
\hline White & $180(57 \%)$ & $179(57 \%)$ \\
\hline Black & $114(36 \%)$ & $112(36 \%)$ \\
\hline Asian & $6(2 \%)$ & $10(3 \%)$ \\
\hline \multicolumn{3}{|l|}{ Ethnicity* } \\
\hline Hispanic or Latino & $72(23 \%)$ & $65(21 \%)$ \\
\hline Not Hispanic or Latino & $240(77 \%)$ & $249(79 \%)$ \\
\hline \multicolumn{3}{|l|}{ HIV disease status } \\
\hline Asymptomatic & $286(91 \%)$ & $286(91 \%)$ \\
\hline Symptomatic & $16(5 \%)$ & $14(4 \%)$ \\
\hline AIDS & $12(4 \%)$ & $15(5 \%)$ \\
\hline \multicolumn{3}{|l|}{ HIV risk factor† } \\
\hline Heterosexual sex & $61(19 \%)$ & $62(20 \%)$ \\
\hline Homosexual sex & $251(80 \%)$ & $250(79 \%)$ \\
\hline Intravenous drug use & $5(2 \%)$ & $4(1 \%)$ \\
\hline HIV-1 RNA, $\log _{10}$ copies per $\mathrm{mL}$ & $4.42(4.03-4 \cdot 87)$ & $4 \cdot 51(4 \cdot 04-4 \cdot 87)$ \\
\hline \multicolumn{3}{|c|}{ HIV-1 RNA concentration category, copies per mL } \\
\hline$\leq 100000$ & $261(83 \%)$ & $265(84 \%)$ \\
\hline$>100000$ & $53(17 \%)$ & $50(16 \%)$ \\
\hline $\begin{array}{l}\text { Median CD4 count, } \\
\text { cells per } \mu \mathrm{L}\end{array}$ & $443(299-590)$ & $450(324-608)$ \\
\hline \multicolumn{3}{|c|}{ CD4 cell count category, cells per $\mu \mathrm{L}$} \\
\hline$<200$ & $36(11 \%)$ & $32(10 \%)$ \\
\hline 200-499 & $156(50 \%)$ & $149(47 \%)$ \\
\hline$\geq 500$ & $122(39 \%)$ & $134(43 \%)$ \\
\hline $\begin{array}{l}\text { Creatinine clearance by } \\
\text { Cockcroft-Gault formula, } \\
\mathrm{mL} / \mathrm{min}\end{array}$ & $126(108-146)$ & $123(107-144)$ \\
\hline Body-mass index, $\mathrm{kg} / \mathrm{m}^{2}$ & $25 \cdot 1(22 \cdot 4-28 \cdot 7)$ & $24 \cdot 9(22 \cdot 5-29 \cdot 1)$ \\
\hline \multicolumn{3}{|c|}{ Primary resistance-associated mutations } \\
\hline INSTI & $4(1 \%)$ & $4(1 \%)$ \\
\hline NRTIS & $6(2 \%)$ & $5(2 \%)$ \\
\hline NNRTIף & $36(11 \%)$ & $51(16 \%)$ \\
\hline Protease inhibitor\| & $12(4 \%)$ & $11(3 \%)$ \\
\hline
\end{tabular}

Data are median (IQR) or $\mathrm{n}(\%)$. INSTI=integrase strand transfer inhibitor. $\mathrm{NRTI}=$ nucleoside reverse transcriptase inhibitor. NNRTI=non-nucleoside reverse transcriptase inhibitor. *Collection of race and ethnicity data was not permitted for two participants in the bictegravir group and one in the dolutegravir group per local regulations. $† A$ participant may fit more than one HIV risk factor category. ‡Thr66Ala/lle/Lys, Glu92Gly/Gln, Thr97Ala, Phe121Tyr, Tyr143Cys/His/Arg, Ser147Gly, Gln148His/Lys/Arg, Asn155His/Ser, Arg263Lys. SMet41Leu, Lys65Glu/Asn/Arg, Asp67Asn, Thr69 insertions, Lys70Glu/Arg, Leu74lle/Val, Tyr115Phe, Gln151Met, Met184Val/lle, Leu210Trp, Thr215Tyr/Phe, Lys219Glu/Asn/Gln/Arg. ILeu100lle, Lys101Glu/Pro, Lys103Asn/Ser, Val106Ala/Met, Val108lle, Glu138Ala/Gly/Lys/Gln/Arg, Val179Leu, Tyr181Cys/lle/Val, Tyr188Cys/Leu/His, Gly190Ala/Glu/Gln/Ser, His221Tyr, Pro225His, Phe227Cys, Met230lle/Leu. ||Asp30Asn, Val32lle, Met46lle/Leu, Ile47Ala/Val, Gly48Val, Ile50Val/Leu, Ile54Leu/Met, Gln58Glu, Thr74Pro, Leu76Val, Val82Ala/Phe/Leu/Thr/Ser, Asn83Asp, lle84Val, Asn88Ser, Leu90Met.

Table 1: Baseline demographic and clinical characteristics 
including treatment group as a fixed-effect in the model. No adjustments were made for multiple comparison; all $\mathrm{p}$ values for secondary outcomes were exploratory.

We used SAS software version 9.4 (SAS Institute Inc, Cary, NC, USA) for all analyses. We assessed non-inferiority with a conventional 95\% CI approach for the difference in proportion of patients with a virological response (bictegravir group minus dolutegravir group) with a prespecified $n$ on-inferiority $m$ argin o $f-12 \%$, b ased o $n$ published US FDA regulatory guidance. ${ }^{?}$

This study was done according to protocol without substantial deviations and is registered with ClinicalTrials. gov, number NCT02607930.

\section{Role of the funding source}

The funder of the study had the lead role in study design, data collection, data analysis, data interpretation and, along with DAW, writing of the manuscript. The corresponding author had full access to all the data in the study and had final $r$ esponsibility $f$ or $t$ he $d$ ecision $t$ o $s$ ubmit $f$ or publication.

\section{Results}

Between Nov 13, 2015, and July 14, 2016, 739 participants were screened for this study, of whom 108 were excluded and 631 were enrolled. 316 participants were assigned to the bictegravir group and 315 to the dolutegravir group (figure 1 ). $\mathrm{O} \mathrm{ft}$ hese, $314 \mathrm{r}$ eceived a $\mathrm{t} l$ east o ne $\mathrm{d}$ ose of bictegravir, emtricitabine, and tenofovir alafenamide and 315 of dolutegravir, abacavir, and lamivudine. Demographics and baseline characteristics were balanced between the two treatment groups (table 1).

At 96 weeks, 276 (88\%) of 314 participants in the bictegravir group had plasma HIV-1 RNA less than 50 copies per $\mathrm{mL}$ compared with $283(90 \%)$ of 315 in the dolutegravir group (difference $-1 \cdot 9 \%, 95 \% \mathrm{CI}-6.9$ to $3 \cdot 1$; table 2, figure 2); therefore, the bictegravir regimen was non-inferior to the dolutegravir regimen in the US FDA snapshot analysis. Two (1\%) participants in the bictegravir group and seven $(2 \%)$ in the dolutegravir group had HIV-1 RNA of 50 copies per mL or more at week 96 or when last tested. Differences existed between treatment groups in the subgroups with cumulative adherence less than $95 \%$ and those who were older than 50 years (appendix p 7). In both cases, the difference was driven by participants who did not have available data in the analysis window and whose last on-treatment assessment of HIV-1 RNA was less than 50 copies per mL (appendix pp 5-6), rather than any evidence of virological failure. No significant $d$ ifferences we re de tected be tween th $e$ two treatments in the other subgroups and no interactions between treatment and subgroup for other prespecified subgroups, including baseline viral load and CD4 strata (appendix p 7).

The proportion of participants with HIV-1 RNA less than 20 copies per $\mathrm{mL}$ at week 96 by FDA snapshot algorithm was $262(83 \%)$ of 314 participants in the

\begin{tabular}{|c|c|c|c|}
\hline & $\begin{array}{l}\text { Bictegravir, } \\
\text { emtricitabine, } \\
\text { and tenofovir } \\
\text { alafenamide } \\
\text { group }(n=314)\end{array}$ & $\begin{array}{l}\text { Dolutegravir, } \\
\text { abacavir, } \\
\text { and lamivudine } \\
\text { group }(n=315)\end{array}$ & $\begin{array}{l}\text { Percentage } \\
\text { difference* }^{*}\end{array}$ \\
\hline HIV-1 RNA $<50$ copies per $\mathrm{mL}$ & $276(88 \%)$ & $283(90 \%)$ & $-1.9 \%(-6.9$ to 3.1$)$ \\
\hline HIV-1 RNA $\geq 50$ copies per $\mathrm{mL}$ & $2(1 \%)$ & $7(2 \%)$ & .. \\
\hline $\begin{array}{l}\text { HIV1 RNA } \geq 50 \text { copies per } m L \text { at week } 96 \\
\text { window }\end{array}$ & $1(<1 \%)$ & $3(1 \%)$ & .. \\
\hline Discontinued because of no efficacy & 0 & 0 & .. \\
\hline $\begin{array}{l}\text { Discontinued because of other reasons } \uparrow \text { and } \\
\text { last available HIV-1 RNA } \geq 50 \text { copies per } \mathrm{mL}\end{array}$ & $1(<1 \%)$ & $4(1 \%)$ & .. \\
\hline No virological data at week 96 window & $36(11 \%)$ & $25(8 \%)$ & .. \\
\hline $\begin{array}{l}\text { Discontinued because of adverse event or } \\
\text { death } \neq\end{array}$ & $1(<1 \%)$ & $5(2 \%)$ & .. \\
\hline $\begin{array}{l}\text { Discontinued because of other reasons } † \text { and } \\
\text { last available HIV-1 RNA }<50 \text { copies per } \mathrm{mL}\end{array}$ & $32(10 \%)$ & $17(5 \%)$ & .. \\
\hline Missing data but on study drug & $3(1 \%)$ & $3(1 \%)$ & .. \\
\hline $\begin{array}{l}\text { HIV-1 RNA }<50 \text { copies per } \mathrm{mL} \text { by missing as } \\
\text { failure } \$\end{array}$ & $276 / 314(88 \%)$ & $286 / 315(91 \%)$ & $-2 \cdot 9 \%(-7 \cdot 8$ to $2 \cdot 1)$ \\
\hline $\begin{array}{l}\text { HIV-1 RNA }<50 \text { copies per } \mathrm{mL} \text { by missing as } \\
\text { excluded } \$\end{array}$ & $276 / 279(99 \%)$ & $286 / 288(99 \%)$ & $-0 \cdot 4 \%(-2 \cdot 5$ to $1 \cdot 7)$ \\
\hline HIV-1 RNA <20 copies per mL & $262 / 314(83 \%)$ & $267 / 315(85 \%)$ & $-1 \cdot 2 \%(-6 \cdot 9$ to $4 \cdot 6)$ \\
\hline $\begin{array}{l}\text { HIV-1 RNA }<50 \text { copies per } \mathrm{mL} \text { by per-protocol } \\
\text { analysis }\end{array}$ & $275 / 276(>99 \%)$ & $278 / 281(99 \%)$ & $0 \cdot 7 \%(-1 \cdot 3$ to $2 \cdot 7)$ \\
\hline
\end{tabular}

Data are $\mathrm{n}(\%)$ and $\%(95 \% \mathrm{Cl})$. The week 96 window is between days 631 and 714 (inclusive). ${ }^{*}$ The difference in percentages of participants with HIV-1 RNA $<50$ copies per $\mathrm{mL}$ between treatment groups and its $95 \% \mathrm{Cl}$ were calculated based on the Mantel-Haenszel proportions adjusted by baseline HIV-1 RNA stratum and region stratum. †Other reasons include investigator's decision, participant decision, lost to follow-up, non-compliance with study drug, protocol violation, pregnancy, and study termination by funder. ¥One death in the bictegravir, emtricitabine, and tenofovir alafenamide occurred after the participant achieved the week 96 outcome. SDifference in percentages and $95 \%$ Cls are based on a dichotomised response: HIV- 1 RNA $<50$ copies per $\mathrm{mL}$ versus HIV- 1 RNA $\geq 50$ copies per mL; patients with missing HIV-1 RNA at week 96 were considered $\geq 50$ copies per $\mathrm{mL}$ for missing as failure approach and were excluded for missing as excluded approach.

Table 2: Virological outcomes at week 96

bictegravir group and 267 (85\%) of 315 in the dolutegravir group (percentage difference $-1 \cdot 2 \%, 95 \% \mathrm{CI}-6 \cdot 9$ to $4 \cdot 6$ ). In the per-protocol analysis, the proportion of participants with HIV-1 RNA less than 50 copies per mL was high in both treatment groups: 275 (>99\%) of 276 for bictegravir, emtricitabine, and tenofovir alafenamide and 278 (99\%) of 281 for dolutegravir, abacavir, and lamivudine (difference $0 \cdot 7 \%, 95 \% \mathrm{CI}-1 \cdot 3$ to $2 \cdot 7$ ). Results from the missing as failure and missing as excluded analyses were consistent with the main analysis (table 2). CD4 cell count increased in both treatment groups, with mean changes from baseline at week 96 of 287 (SD 207) cells per $\mu \mathrm{L}$ in the bictegravir group and 288 (247) cells per $\mu \mathrm{L}$ in the dolutegravir group.

Five participants with protocol-defined criteria for resistance testing were included in the week 96 resistance analysis population; all were in the dolutegravir, abacavir, and lamivudine group. One discontinued study drug before week 48 , two had resistance testing before week 48 and again met criteria and discontinued study drug between weeks 48 and 96, and two met criteria and discontinued between weeks 48 and 96 . One participant in 

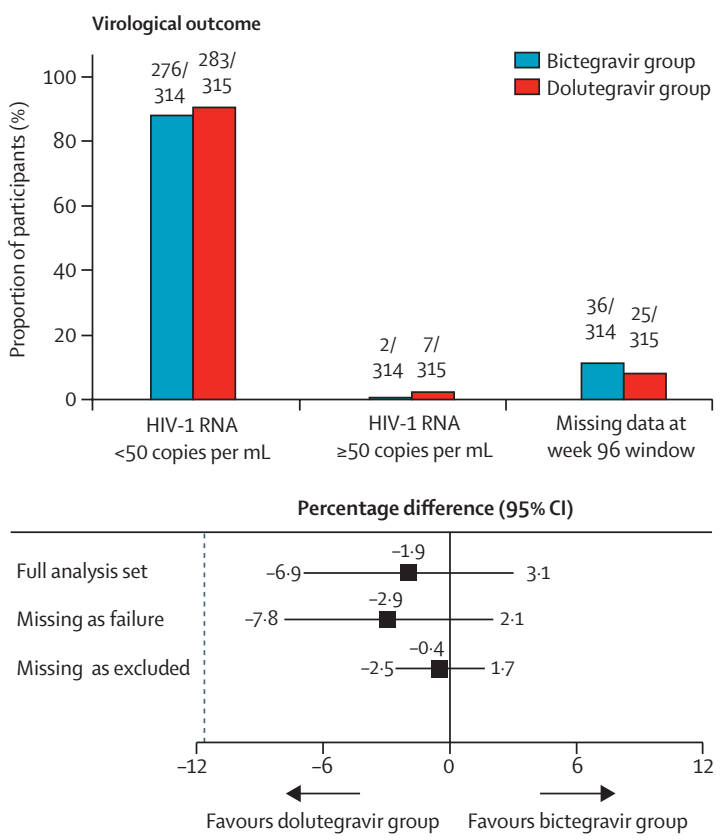

Figure 2: Virological outcomes at week 96

Bictegravir group=bictegravir, emtricitabine, and tenofovir alafenamide group. Dolutegravir group=dolutegravir, abacavir, and lamivudine group.

the bictegravir group who met inclusion criteria for resistance analysis at week 48 achieved HIV-1 RNA of less than 50 copies per $\mathrm{mL}$ on study drug and, therefore, is not included in the resistance analysis population at week 96 . No emergent resistance developed to any component of either treatment regimen. Baseline primary nucleoside reverse transcriptase inhibitor (NRTI) resistance-associated mutations were present in $11(2 \%)$ participants and consisted of Tyr115Phe or thymidine analogue mutations. ${ }^{10}$ Baseline integrase genotyping data was assessed retrospectively by deep sequencing and analysed at mutation frequencies of at least 15\%. Pretreatment INSTI resistanceassociated mutations were present in eight (1\%) participants; one had Gln148His and Gly140Ser substitutions at greater than $99 \%$ frequency and seven had Thr97Ala. The presence of NRTI or INSTI resistance-associated mutations did not impact treatment outcomes. Notably, the participant with Gln148His and Gly140Ser (confirmed by population Sanger sequencing, with phenotypic resistance to raltegravir and elvitegravir, partial sensitivity to dolutegravir, and full sensitivity to bictegravir) was in the bictegravir group and had HIV-1 RNA less than 50 copies per mL at week 96.

Both treatments were generally well tolerated, with most adverse events reported as mild or moderate in severity. The most common adverse events were nausea, which was more common in the dolutegravir group than the bictegravir group $(\mathrm{p}=0 \cdot 0001)$, diarrhoea, and headache (table 3). Prevalence and incidence of nausea declined over the 96-week study period (appendix p 10). No participants in the bictegravir group had adverse events that led to

\begin{tabular}{|c|c|c|}
\hline & $\begin{array}{l}\text { Bictegravir, } \\
\text { emtricitabine, } \\
\text { and tenofovir } \\
\text { alafenamide } \\
\text { group }(n=314)\end{array}$ & $\begin{array}{l}\text { Dolutegravir, } \\
\text { abacavir, } \\
\text { and lamivudine } \\
\text { group }(n=315)\end{array}$ \\
\hline All adverse events & $292(93 \%)$ & $302(96 \%)$ \\
\hline \multicolumn{3}{|c|}{ Adverse event in at least $10 \%$ of participants } \\
\hline Nausea & $36(11 \%)$ & $76(24 \%)$ \\
\hline Diarrhoea & $48(15 \%)$ & $50(16 \%)$ \\
\hline Headache & $41(13 \%)$ & $51(16 \%)$ \\
\hline Upper respiratory tract infection & $33(11 \%)$ & $51(16 \%)$ \\
\hline Nasopharyngitis & $36(11 \%)$ & $39(12 \%)$ \\
\hline Syphilis & $27(9 \%)$ & $39(12 \%)$ \\
\hline Fatigue & $27(9 \%)$ & $35(11 \%)$ \\
\hline Back pain & $25(8 \%)$ & $30(10 \%)$ \\
\hline Insomnia & $22(7 \%)$ & $31(10 \%)$ \\
\hline Grade 3 or 4 adverse events & $42(13 \%)$ & $37(12 \%)$ \\
\hline Serious adverse events & $36(11 \%)$ & $39(12 \%)$ \\
\hline Study drug-related adverse events & $89(28 \%)$ & $127(40 \%)$ \\
\hline \multicolumn{3}{|c|}{ Study drug-related adverse events in at least $5 \%$ of participants } \\
\hline Nausea & $18(6 \%)$ & $55(17 \%)$ \\
\hline Diarrhoea & $19(6 \%)$ & $13(4 \%)$ \\
\hline Headache & $16(5 \%)$ & $16(5 \%)$ \\
\hline $\begin{array}{l}\text { Study drug-related serious adverse } \\
\text { events }\end{array}$ & $3(1 \%)^{*}$ & $1(<1 \%)$ \\
\hline $\begin{array}{l}\text { Any adverse events leading to study } \\
\text { drug discontinuation }\end{array}$ & 0 & $5(2 \%)$ \\
\hline Death & $2(1 \%)$ & 0 \\
\hline
\end{tabular}

Data are $\mathrm{n}(\%) .{ }^{*}$ One of these was reported as related at the time of analysis and was subsequently updated to unrelated when additional information was available to the investigator.

Table 3: Adverse events through week 96

study drug discontinuation, whereas five (2\%) participants in the dolutegravir group did. No neuropsychiatric adverse events met the more than 5\% difference in incidence threshold for statistical testing. However, the frequency of dizziness and sleep disorder (MedDRA preferred terms) were two times higher in the dolutegravir group than in the bictegravir group (18 [6\%] of 315 vs nine [3\%] of 314 for dizziness and 13 [4\%] vs three [1\%] for sleep disorder). $36(11 \%)$ participants in the bictegravir group versus $39(12 \%)$ participants in the dolutegravir group had a serious adverse event. Five participants had adverse events that led to study drug discontinuation in the dolutegravir group: one nausea and generalised rash (day 4); one thrombocytopenia (day 50); one chronic pancreatitis and steatorrhoea (day 134); one depression (day 248); and one renal failure (day 621). All except the renal failure were considered by the investigator to be related to study drugs; all were grade 3 except one nausea and one depression, which were grade 2 (table 3 ). Participants in the bictegravir group had a lower incidence of drug-related adverse events than did those in the dolutegravir group (89 [28\%] of $314 \mathrm{vs}$ 127 [40\%] of $315, \mathrm{p}=0 \cdot 002$ ); these events were primarily mild or moderate in severity and most occurred before 
week 48. The difference between groups was driven mainly by the significant difference in drug-related nausea which occurred in $18(6 \%)$ of 314 in the bictegravir group versus $55(17 \%)$ of 315 in the dolutegravir group $(\mathrm{p}<0 \cdot 0001)$. Study drug-related serious adverse events were originally reported for three participants in the bictegravir group (one sudden death, one generalised tonic-clonic seizure, and one spontaneous abortion) versus one patient in the dolutegravir group (gastroenteritis, steatorrhoea, and acute pancreatitis). One event in the bictegravir group (sudden death) was subsequently attributed to suicide and not related to study drug. Adverse events were similar to those reported at week 48 , with no new safety findings between weeks 48 and 96 for either treatment group. No abnormal electrocardiogram findings w ere a ssociated w ith e ither treatment.

Between weeks 48 and 96, two deaths occurred, both in the bictegravir group. These events were recreational drug overdose (one) and suicide (one; in a participant with ongoing substance abuse); both events were considered unrelated to study treatment.

Three women had confirmed pregnancies w hile o $n$ study, two in the bictegravir group and one in the dolutegravir group. One of the two participants in the bictegravir group was taken off s tudy treatments when pregnancy was confirmed a nd s ubsequently delivered a healthy full-term infant. The other woman in the bictegravir group had a spontaneous abortion at an estimated 2 weeks' gestation; the participant recovered from the event without complications. The pregnancy in the dolutegravir group was terminated with an elective abortion and study drugs were continued.

One or more grade 3 or 4 laboratory abnormalities were reported for $71(23 \%)$ of 314 participants in the bictegravir group and 62 (20\%) of 315 in the dolutegravir group; incidence and types of abnormalities were generally balanced between treatment groups (appendix p 8). Most abnormalities were transient and resolved on therapy. The overall laboratory safety profiles were similar to those observed at week 48. No proximal tubulopathy or Fanconi syndrome were reported in either group. No study participant in the bictegravir group discontinued because of a renal adverse event. One individual in the dolutegravir group with a medical history of poorly controlled diabetes and hypertension discontinued treatment because of an adverse event of renal failure on day 621. Small increases from baseline in median serum creatinine and decreases in eGFR occurred at week 96 for both groups (table 4). At 96 weeks, percentage changes in quantitative proteinuria (total urinary albumin-tourine creatinine ratio) and tubular proteinuria (retinol binding protein and $\beta 2$-microglobulin-to-urine creatinine ratios) were also similar between groups (table 4).

Changes from baseline in fasting high-density lipoprotein and triglycerides were similar between groups at week 96 (appendix p 9). Significant differences

\begin{tabular}{|c|c|c|c|c|c|}
\hline & \multicolumn{2}{|c|}{$\begin{array}{l}\text { Bictegravir, emtricitabine, } \\
\text { and tenofovir alafenamide } \\
\text { group }(n=314)\end{array}$} & \multicolumn{2}{|c|}{$\begin{array}{l}\text { Dolutegravir, abacavir, } \\
\text { and lamivudine group } \\
(\mathrm{n}=315)\end{array}$} & \multirow[t]{2}{*}{$\mathrm{p}$ value } \\
\hline & $\mathrm{n}$ & Median (IQR) & $\mathrm{n}$ & Median (IQR) & \\
\hline \multicolumn{6}{|c|}{ Serum creatinine, $\mathrm{mg} / \mathrm{dL}$} \\
\hline Baseline & 314 & $0.90(0.80$ to 1.00$)$ & 315 & 0.91 (0.81 to 0.99$)$ & 0.92 \\
\hline Change at week 96 & 277 & 0.08 (0.01 to 0.15$)$ & 286 & $0.09(0.03$ to 0.17$)$ & 0.067 \\
\hline \multicolumn{6}{|c|}{ Estimated glomerular filtration rate, ${ }^{*} \mathrm{~mL} / \mathrm{min}$} \\
\hline Baseline & 314 & $125 \cdot 9(107 \cdot 7$ to $146 \cdot 3)$ & 315 & $123 \cdot 0(107 \cdot 0$ to $144 \cdot 3)$ & 0.76 \\
\hline Change at week 96 & 277 & $-7 \cdot 8(-16 \cdot 4$ to $3 \cdot 6)$ & 286 & $-9 \cdot 6(-19 \cdot 9$ to $-0 \cdot 4)$ & 0.01 \\
\hline \multicolumn{6}{|c|}{ Urine albumin-to-creatinine ratio, $\mathrm{mg} / \mathrm{g}$} \\
\hline Baseline & 309 & $5 \cdot 5(3 \cdot 7$ to $9 \cdot 2)$ & 312 & $5 \cdot 4(3 \cdot 7$ to $9 \cdot 1)$ & 0.72 \\
\hline $\begin{array}{l}\text { Percentage change at } \\
\text { week } 96\end{array}$ & 272 & $-0 \cdot 3(-33 \cdot 5$ to $59 \cdot 6)$ & 284 & $5 \cdot 2(-25 \cdot 9$ to $57 \cdot 0)$ & 0.25 \\
\hline \multicolumn{6}{|c|}{ Urine $\beta 2$-microglobulin-to-creatinine ratio, $\mu \mathrm{g} / \mathrm{g}$} \\
\hline Baseline & 307 & $108 \cdot 1$ (71.7 to $184 \cdot 4)$ & 311 & $109.8(77.6$ to $191 \cdot 8)$ & 0.92 \\
\hline $\begin{array}{l}\text { Percentage change at } \\
\text { week } 96\end{array}$ & 271 & $-30 \cdot 8(-58 \cdot 1$ to $20 \cdot 9)$ & 283 & $-29 \cdot 4(-57 \cdot 6$ to $12 \cdot 1)$ & 0.96 \\
\hline \multicolumn{6}{|c|}{ Urine retinol binding protein-to-creatinine ratio, $\mu \mathrm{g} / \mathrm{g}$} \\
\hline Baseline & 308 & $81 \cdot 0(58 \cdot 3$ to $122 \cdot 4)$ & 312 & $83.7(59.8$ to $120 \cdot 4)$ & 0.55 \\
\hline $\begin{array}{l}\text { Percentage change at } \\
\text { week } 96\end{array}$ & 272 & $21 \cdot 2(-12 \cdot 2$ to $66 \cdot 5)$ & 284 & $22 \cdot 1(-12.8$ to 78.7$)$ & 0.91 \\
\hline
\end{tabular}

Table 4: Changes in quantitative measures of proteinuria from baseline at week 96

were measured in the median changes from baseline in fasting total cholesterol, low-density lipoprotein, and total cholesterol-to-high-density lipoprotein ratio at week 96 (appendix p 9). Initiation of lipid-modifying drugs during the study through week 96 was not different between the groups.

There were small changes from baseline in hip and lumbar spine bone mineral density that were similar between the two groups; mean percentage changes at week 96 in hip density were $-1.13 \%$ (SD $2 \cdot 77$ ) in the bictegravir group versus $-1.26 \%(2.85)$ in the dolutegravir group $(\mathrm{p}=0.59)$ and changes in lumbar spine density were $-0.71 \%(3.87)$ versus $-0.22 \%(3.52$; $\mathrm{p}=0 \cdot 14$; figure 3 ). Weight increases after treatment initiation occurred in both groups. The median change was $3.6 \mathrm{~kg}$ (IQR $0.0-8.5)$ in the bictegravir group and $2.4 \mathrm{~kg}(-0.4$ to 5.8$)$ in the dolutegravir group.

\section{Discussion}

Maximal and durable suppression of plasma HIV-1 RNA as well as the enhancement of quality of life are among the major goals of treatment of HIV-1 infection. ${ }^{2}$ Here, we report data showing non-inferiority of coformulated bictegravir, emtricitabine, and tenofovir alafenamide over co-formulated dolutegravir, abacavir, and lamivudine at 96 weeks after initiation in treatmentnaive people living with HIV-1 infection. Viral rebound was rare throughout the study. Overall, these virological outcomes data are consistent with those reported at week $48 .^{6}$ 

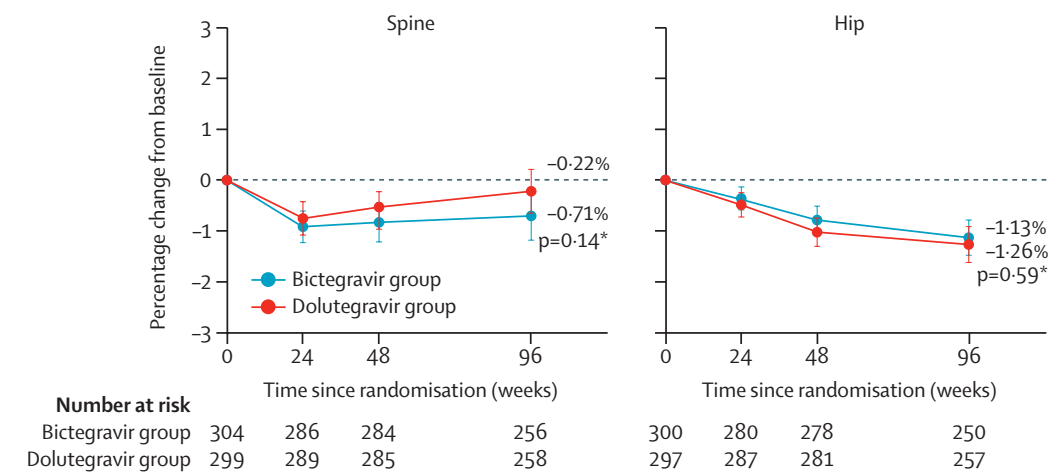

Figure 3: Percentage change from baseline at weeks 24,48 , and 96 in lumbar spine and hip bone mineral density by dual-energy $\mathrm{x}$-ray absorptiometry

Data points are means, error bars are $95 \%$ Cls. ${ }^{*} p$ values are at week 96 by analysis of variance.

As expected, given the inhibition of renal creatinine transporters by both bictegravir and dolutegravir, both groups had small median increases from baseline in serum creatinine and decreases in eGFR that occurred early and were sustained..$^{11}$ Likewise, small but similar decreases in bone mineral density occurred in both study groups. Larger changes from baseline in total cholesterol and direct low-density lipoprotein cholesterol occurred in participants in the bictegravir group. Use of lipid-lowering therapy during the trial was low in both study groups, suggesting the clinical relevance of this $10 \mathrm{mg} / \mathrm{dL}$ difference between the two groups was minimal. The greater increase in low-density lipoprotein cholesterol in the bictegravir group was unexpected given previously reported week 48 data on the lipid profiles of both study regimens. Notably, in a study of treatment-naive individuals initiating bictegravir, emtricitabine, and tenofovir alafenamide versus dolutegravir, emtricitabine, and tenofovir alafenamide, significant differences in the median change in lipid concentrations at 96 weeks, including low-density lipoprotein cholesterol, were not observed. ${ }^{12}$

Several observational studies have described differential increases in weight after the initiation of or switch to different classes of antiretrovirals. ${ }^{13-15}$ Some, but not all, of these reports suggest that weight gain is greater among those treated with INSTIs. ${ }^{16-18}$ As expected for a treatmentnaive trial, median increases from baseline in weight were observed in both study groups. Longer-term trends in weight and the factors associated with changes from baseline will be examined in future planned analyses.

Overall, these results show that both regimens were efficacious and well tolerated. In distinguishing between the two, the higher frequency of investigatorreported and patient-reported nausea should be considered. Additionally, the limitations of the use of dolutegravir, abacavir, and lamivudine, including among those with hepatitis B virus co-infection or with an eGFR less than $50 \mathrm{~mL} / \mathrm{min}$, as well as the requirement for HLA-B*5701 screening, might favour the use of bictegravir, emtricitabine, and tenofovir alafenamide in some individuals.

As described previously, there are limitations to this investigation that should be considered when interpreting these data. Foremost, the proportion of women enrolled in the trial was low. However, in a study that enrolled only women $(n=470)$ with suppressed plasma HIV-1 RNA who were randomly assigned either to continue prestudy antiretroviral therapy or to switch to bictegravir, emtricitabine, and tenofovir alafenamide, more than $95 \%$ of the participants in each treatment group maintained viral suppression at week 48 with no discontinuations of study drug due to adverse events. ${ }^{19}$ Neuropsychiatric adverse effects of INSTI-containing regimens have been previously reported; however, each individual adverse effect is described as relatively uncommon..$^{20-22}$ Therefore, this trial probably had too few participants to detect differences between the regimens in any neuropsychiatric adverse event as reported by investigators. Furthermore, although 96-week data provide a valuable assessment of the durability of the efficacy and safety of these regimens, the need for HIV therapy remains lifelong. Longer-term data is needed to provide insights into the advantages and disadvantages of these therapeutic options. Participants in this study will continue to be followed up until week 144 .

In summary, as was observed at week 48 , bictegravir, emtricitabine, and tenofovir alafenamide after 96 weeks of therapy was non-inferior to dolutegravir, abacavir, and lamivudine, with no emergent drug resistance or tubulopathy detected, but with a better gastrointestinal tolerability profile. Bictegravir, emtricitabine, and tenofovir alafenamide can be used in those co-infected with hepatitis B virus and eGFRs as low as $30 \mathrm{~mL} / \mathrm{min}$ and does not require HLA-B*5701 screening. Therefore, bictegravir, emtricitabine, and tenofovir alafenamide is a potent and well tolerated option for people with HIV-1 infection initiating treatment.

\section{Contributors}

DAW, YY, AB, AC, MAT, CB, DH, MNR, and AA enrolled participants. $\mathrm{XW}$ and HM designed the study. XW and RA did the data analyses, which were reviewed and interpreted by DAW, SEC, DB, and HM. The first draft was written by SEC and DAW. All authors reviewed and interpreted analyses of data, contributed edits of the final report, and approved the draft manuscript. DAW and SEC made the decision to submit the manuscript for publication.

\section{Declaration of interests}

DAW reports receiving honoraria for advisory board participation and consultancy from Gilead Sciences, ViiV, Merck, and Janssen; and research grant support from Gilead Sciences, ViiV, Janssen, and Merck and Co. AB has served on speakers bureaus for AbbVie, Bristol-Myers Squibb (BMS), Gilead, and Janssen; and advisory boards for AbbVie, BMS, Gilead, and Merck Sharp \& Dohme (MSD). AC reports receiving conference travel bursaries and consultancy fees from Gilead Sciences and ViiV Healthcare. XW, RA, SEC, DB, and HM are employees of Gilead Sciences and own stock in the company. MAT has received research funding paid to the AIDS Research Consortium of Atlanta from BMS, CytoDyn, GlaxoSmithKline, Gilead, MSD, Roche Laboratories, Taimed, and ViiV. CB reports grants from Gilead Sciences, Braintree, Novo Nordisk, ViiV Healthcare, CoLucid, SlieaGen, Shionogi, Sanofi, Daiichi Sankyo, and Theratechnologies; and personal fees from 
Gilead Sciences and Theratechnologies. MNR has served on the speakers' bureau for Gilead, Janssen, Abbvie, and Allergan; and has consulted for Gilead, Viiv, and Merck. AA has received grants from AbbVie, BMS, Gilead, Merck, ViiV, and Janssen Cilag. All other authors report no competing interests.

\section{Data sharing}

Anonymised individual participant data and study documents can be requested by email (datarequest@gilead.com) for further research.

\section{Acknowledgments}

This study was sponsored by Gilead Sciences, Inc. We thank the individuals who participated in this trial and their families, the principal investigators (appendix pp 1-4), and their staff, the Gilead study staff, and Anna Kido (Gilead employee) for providing editorial assistance. Parts of this study have been presented at IDWeek; San Francisco, CA, USA; Oct 3-7, 2018.

\section{References}

1 European AIDS Clinical Society (EACS). EACS treatment guidelines, version 9.1 updated. Brussels, Belgium: EACS, October 2018. http://www.eacsociety.org/files/2018_ guidelines-9·1-english.pdf (accessed Dec 18, 2018).

2 Panel on Antiretroviral Guidelines for Adults and Adolescents. Guidelines for the use of antiretroviral agents in adults and adolescents living with HIV. Washington, DC, USA: Department of Health and Human Services, Oct 25, 2018. http://www.aidsinfo.nih. gov/ContentFiles/AdultandAdolescentGL.pdf (accessed Dec 18, 2018).

3 Günthard HF, Saag MS, Benson CA, et al. antiretroviral drugs for treatment and prevention of HIV infection in adults: 2016 recommendations of the International Antiviral Society-USA panel. JAMA 2016; 316: 191-210.

4 European Medicines Agency. Triumeq (dolutegravir/abacavir/lamibudie) summary of product characteristics. Middlesex: ViiV Healthcare UK Limited, Dec 3, 2018. https://www.ema.europa.eu/documents/product-information/ triumeq-epar-product-information_en.pdf (accessed Dec 18, 2018).

5 Bictarvy (bictegravir/emtricitabine/tenofovir alafenamide) summary of product characteristics. Carrigtohill, IE: Gilead Sciences Ireland UC, Dec 13, 2018. https://www.ema.europa.eu/documents/productinformation/biktarvy-epar-product-information_en.pdf (accessed Dec 18, 2018)

6 Gallant J, Lazzarin A, Mills A, et al. Bictegravir, emtricitabine, and tenofovir alafenamide versus dolutegravir, abacavir, and lamivudine for initial treatment of HIV-1 infection (GS-US-380-1489): a double-blind, multicentre, phase 3, randomised controlled non-inferiority trial. Lancet 2017; 390: 2063-72.

7 Wohl D, Clarke A, Maggiolo F, et al. Patient-reported symptoms over 48 weeks among participants in randomized, double-blind, phase III non-inferiority trials of adults with HIV on co-formulated bictegravir, emtricitabine, and tenofovir alafenamide versus co-formulated abacavir, dolutegravir, and lamivudine. Patient 2018; 11: 561-73.

8 Smith F, Hammerstrom T, Soon G, et al. A meta-analysis to assess the FDA DAVP's TLOVR algorithm in HIV submissions. Ther Innov Regul Sci 2011; 45: 291-300.
9 US Food and Drug Administration. Human immunodeficiency virus- 1 infection: developing antiretroviral drugs for treatment-guidance for industry, appendix B. Washington, DC, USA: Department of Health and Human Services, November 2015. https://www.fda.gov/ downloads/drugs/guidancecomplianceregulatoryinformation/ guidances/ucm355128.pdf (accessed Dec 18, 2018).

10 Wensing AM, Calvez V, Günthard HF, et al. 2017 Update of the drug resistance mutations in HIV-1. Top Antivir Med 2017; 24: 132-33.

11 Lazerwith SE, Cai R, Chen X, et al. Discovery of GS-9883, an HIV-1 integrase strand transfer inhibitor (INSTI) with improved pharmacokinetics and in vitro resistance profile. American Society for Microbiology; Boston, MA, USA; June 16-20, 2016. Abstract 414

12 Stellbrink H-J, Arribas J, Stephens JL, et al. Phase III randomized, controlled clinical trial of bictegravir coformulated with FTC/TAF in a fixed-dose combination (B/F/TAF) versus dolutegravir (DTG) + F/TAF in treatment-naive HIV-1 positive adults: week 96. J Int AIDS Soc 2018; 21 (suppl 8): 8.

13 Taramasso L, Ricci E, Menzaghi B, et al. Weight gain: a possible side effect of all antiretrovirals. Open Forum Infect Dis 2017; 4: ofx239.

14 Tate T, Willig AL, Willig JH, et al. HIV infection and obesity: where did all the wasting go? Antivir Ther 2012; 17: 1281-89.

15 Lakey W, Yang LY, Yancy W, Chow SC, Hicks C. Short communication: from wasting to obesity: initial antiretrovira therapy and weight gain in HIV-infected persons. AIDS Res Hum Retroviruses 2013; 29: 435-40.

16 Bhagwat P, Ofotokun I, McComsey GA, et al. Predictors of severe weight/body mass index gain following antiretroviral initiation. Conference on Retroviruses and Opportunistic Infections; Seattle, WA, USA; Feb 13-16, 2017. Abstract 695.

17 Norwood J, Turner M, Bofill C, et al. Brief report: weight gain in persons with HIV switched from efavirenz-based to integrase strand transfer inhibitor-based regimens. J Acquir Immune Defic Syndr 2017; 76: 527-31.

18 Menard A, Meddeb L, Tissot-Dupont H, et al. Dolutegravir and weight gain: an unexpected bothering side effect? AIDS 2017; 31: $1499-502$

19 Kityo C, Hagins D, Koenig, et al. Switching to bictegravir/emtricitabine/tenofovir alafenamide (B/F/TAF) in women. Conference on Retroviruses and Opportunistic Infections; Boston, MA, USA; March 4-7, 2018. Abstract 500.

20 Hoffmann C, Welz T, Sabranski M, et al. Higher rates of neuropsychiatric adverse events leading to dolutegravir discontinuation in women and older patients. HIV Med 2017; 18: 56-63.

21 Peñafiel J, de Lazzari E, Padilla M, et al. Tolerability of integrase inhibitors in a real-life setting. J Antimicrob Chemother 2017; 72: 1752-59.

22 Bonfanti P, Madeddu G, Gulminetti R, et al. Discontinuation of treatment and adverse events in an Italian cohort of patients on dolutegravir. AIDS 2017; 31: 455-57. 